\title{
A Proposal for a Linear Calculation of Gait Asymmetry
}

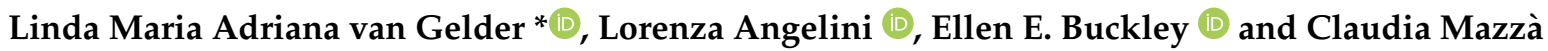 \\ Department of Mechanical Engineering and Insigneo Institute for In Silico Medicine, The University of Sheffield, \\ Sheffield S10 2TN, UK; l.angelini@sheffield.ac.uk (L.A.); e.e.buckley@sheffield.ac.uk (E.E.B.); \\ c.mazza@sheffield.ac.uk (C.M.) \\ * Correspondence: 1.vangelder@sheffield.ac.uk
}

check for

updates

Citation: van Gelder, L.M.A.;

Angelini, L.; Buckley, E.E.; Mazzà, C. A Proposal for a Linear Calculation of Gait Asymmetry. Symmetry 2021, 13, 1560. https://doi.org/10.3390/ sym13091560

Academic Editor: Paul Sung

Received: 13 July 2021

Accepted: 18 August 2021

Published: 25 August 2021

Publisher's Note: MDPI stays neutral with regard to jurisdictional claims in published maps and institutional affiliations.

Copyright: (C) 2021 by the authors. Licensee MDPI, Basel, Switzerland. This article is an open access article distributed under the terms and conditions of the Creative Commons Attribution (CC BY) license (https:// creativecommons.org/licenses/by/ $4.0 /)$.

\begin{abstract}
Pathological gait is often associated with a lack of symmetry. A possible way to quantify this feature is to use acceleration data measured by a sensor located on the lower trunk. The most common approach calculates a symmetry index starting from the autocorrelation function, aiming to measure the divergence in motion of the left and right sides of the body. The various methods proposed to implement this approach are based on nonlinear and discontinuous functions, and the interpretation of their output is far from straightforward. The aim of this study was to propose a linear and easier to interpret quantification measure for gait asymmetry. The proposed measure was tested on data from healthy controls and from patients with Multiple Sclerosis and Parkinson's Disease, and it was shown to negate the flaws present in previous methods and to provide more directly interpretable results.
\end{abstract}

Keywords: asymmetry; gait; accelerometer; IMU

\section{Introduction}

Pathological gait is often associated with a lack of symmetry [1]. Asymmetric gait is less efficient, increases oxygen consumption, and the energy cost of locomotion may lead to loss of bone mass density, osteoarthritis, and musculoskeletal injury [1]. A widely applied approach to quantify asymmetric gait relies on the calculation of autocorrelation from accelerometer data [2,3]. This approach is based on calculations of regularity, which refers to the similarity between consecutive strides or steps. Regularity and symmetry are two metrics that have the potential to explain the quality of gait. These metrics have been shown to carry additional information compared to measures of spatiotemporal symmetry when investigating pathological gait [4]. Recently, regularity and symmetry have been suggested to be potential biomarkers for quantifying gait disability in ageing and diseases, including multiple sclerosis (MS) and Parkinson's disease (PD) [3-5].

Regularity is determined by applying the following unbiased autocorrelation procedure to the measured acceleration signals [3]:

$$
\mathrm{A}_{\text {unbiased }}=\frac{1}{\mathrm{~N}-|\mathrm{m}|} \sum_{\mathrm{i}=1}^{\mathrm{N}-|\mathrm{m}|} \mathrm{x}_{\mathrm{i}} \mathrm{x}_{\mathrm{i}+\mathrm{m}}
$$

where $x_{i}(i=1,2, \ldots, N)$ are timeseries and $m$ is the lag parameter, which is the phase shift in number of samples. Starting from Equation (1), step $\left(\mathrm{Ad}_{1}\right)$ and stride $\left(\mathrm{Ad}_{2}\right)$ regularity are defined as the first two peaks of the correlation between the acceleration signal and the acceleration signal phase-shifted to the average step and stride time (Figure 1). These phase shifts are consistent with the first and second dominant periods of the unbiased autocorrelation curve, respectively. $\mathrm{Ad}_{1}$ and $\mathrm{Ad}_{2}$ can vary between -1 and 1 , with 0 indicating maximum irregularity. When considering lower trunk accelerations, negative values of $\mathrm{Ad}_{1}$ are typically recorded in the medio-lateral (ML) direction as a result of the alternating lower limb motions, while mostly positive values are observed in the anterior- 
posterior (AP) and vertical (V) directions. Positive values of $\mathrm{Ad}_{2}$ are most likely to be observed in all movement directions.

(a)

(b)

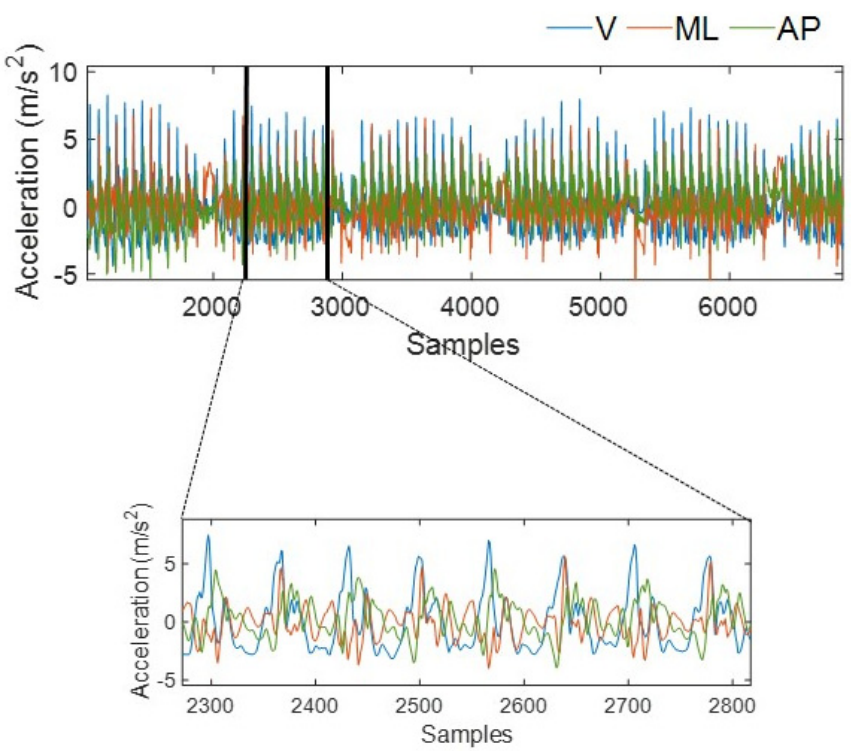

(c)

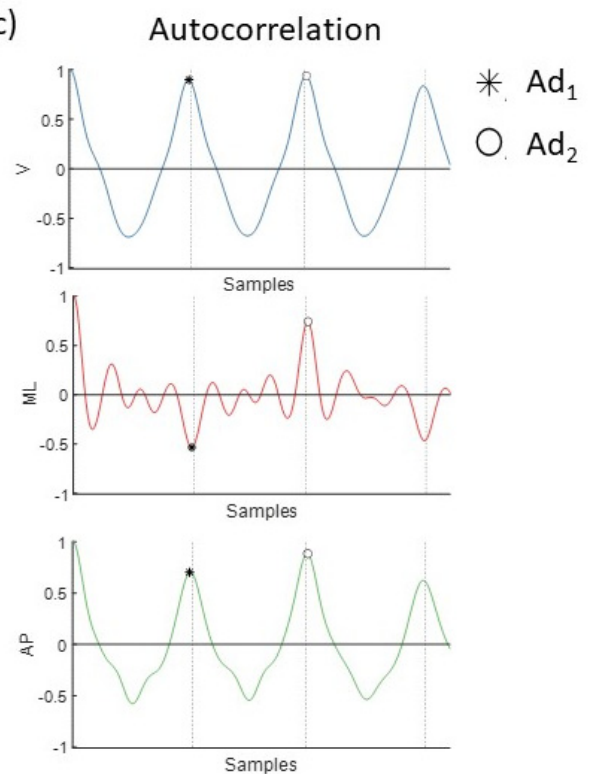

Figure 1. (a) Lower back acceleration signal of one participant walking up and down a $10 \mathrm{~m}$ walkway; (b) Acceleration signal for one pass taken from the signal as displayed in panel a. (c) Step $\left(\operatorname{Ad}_{1}\right)$ and stride $\left(\operatorname{Ad}_{2}\right)$ regularity are defined as the first two peaks of the unbiased autocorrelation coefficient, respectively.

Moe-Nilssen and Helbostad [3] initially proposed to calculate gait symmetry as a ratio between step and stride regularity (Equation (2)). In this definition, perfect theoretical symmetry is achieved when step regularity is equal to the stride regularity, i.e., $\mathrm{S}_{\mathrm{MN}}=1$ in $\mathrm{AP}$ and $\mathrm{V}$ direction and when $\mathrm{S}_{\mathrm{MN}}=-1$ in the ML direction. Values lower or greater than 1 (or -1 in the ML direction) indicate asymmetry in the gait pattern.

$$
\mathrm{S}_{\mathrm{MN}}=\frac{\text { Step regularity }}{\text { Stride regularity }}=\frac{\mathrm{Ad}_{1}}{\mathrm{Ad}_{2}}
$$

Later, Kobsar et al. [2] suggested quantifying gait symmetry using the ratio between the absolute difference between $\mathrm{Ad}_{1}$ and $\mathrm{Ad}_{2}$ and the average of $\mathrm{Ad}_{1}$ and $\mathrm{Ad}_{2}$ (Equation (3)) in order to focus on the actual difference between steps and strides regularity rather than only on their ratio. With this definition, quantifying asymmetry rather than symmetry, greater values indicate a higher degree of asymmetry between the left and right steps, with a value of 0 indicating perfect symmetry, irrespectively of the direction of motion.

$$
\mathrm{AS}_{\mathrm{K}}=\frac{\left|\mathrm{Ad}_{1}-\mathrm{Ad}_{2}\right|}{\operatorname{Mean}\left(\mathrm{Ad}_{1}, \mathrm{Ad}_{2}\right)}
$$

In practice, for both Equations (2) and (3), the value of symmetry tends to infinity as the denominator approaches zero. This generates a non-linear scale and adds complexity to the interpretation of the calculated values. Furthermore, the function surfaces for both $\mathrm{AS}_{\mathrm{K}}$ and $\mathrm{S}_{\mathrm{MN}}$ are discontinuous (Figure $2 \mathrm{a}, \mathrm{b}$ ). This latter point is particularly critical for $\mathrm{AS}_{\mathrm{K}}$ : for two participants with a very similar $\mathrm{Ad}_{1}$ (e.g., -0.71 vs. -0.69 ) and identical $\mathrm{Ad}_{2}$ (e.g., 0.7), the asymmetry calculation might result in very different values (e.g., -282 and 278 for $\mathrm{AS}_{\mathrm{K}}$ in this example). Overall, both definitions of symmetry hinder clinical interpretation of the data and comparison of different outcomes both between and within participants. The aim of this paper is therefore to propose a linear definition of symmetry, illustrating how this can overcome the above discussed issues, leading to results that would be easier to interpret when adopted in a clinical context. 


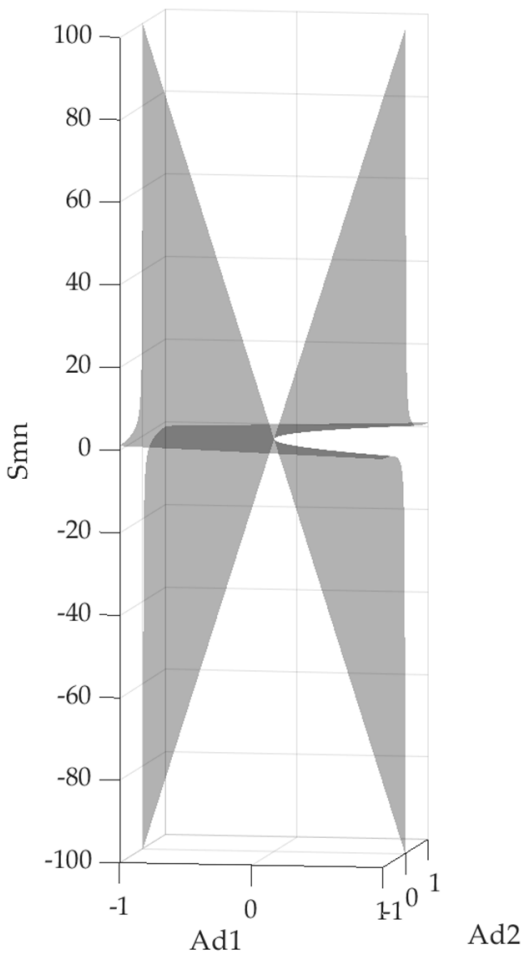

(a)

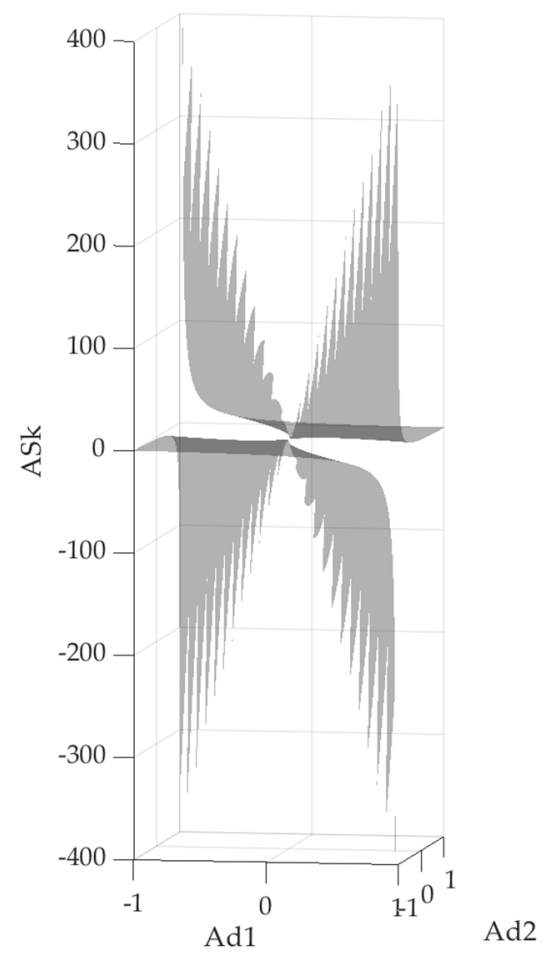

(b)

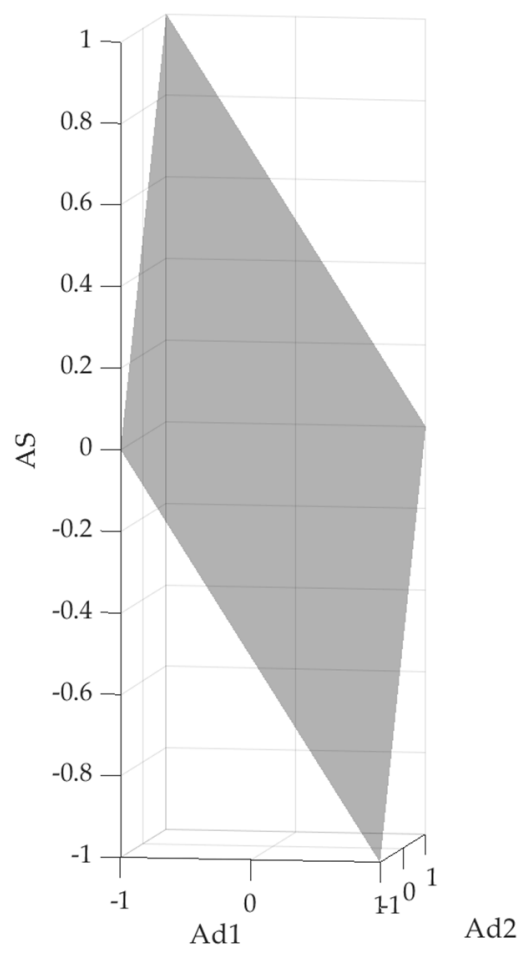

(c)

Figure 2. (a) Function surface of symmetry based on the formula of Moe-Nilssen and Helbostad [3]; (b) Function surface of asymmetry based on the formula of Kobsar et al. [2]. (c) Function surface of asymmetry based on the formula proposed in this article. For the sake of allowing visual representation, all function domains were limited varying $\mathrm{Ad}_{1}$ and $\mathrm{Ad}_{2}$ between -1 and 1 at 0.01 intervals.

\section{Materials and Methods}

To tackle the issues of nonlinearity and discontinuity in the previous equations, we propose an alternative equation for the calculation of asymmetry (AS) in the three directions of motion:

$$
\begin{aligned}
\mathrm{AS}_{\mathrm{AP}} & =\frac{\mathrm{Ad}_{2 \mathrm{AP}}-\mathrm{Ad}_{1 \mathrm{AP}}}{2} \\
\mathrm{AS}_{\mathrm{V}} & =\frac{\mathrm{Ad}_{2 \mathrm{~V}}-\mathrm{Ad}_{1 \mathrm{~V}}}{2} \\
\mathrm{AS}_{\mathrm{ML}} & =\frac{\mathrm{Ad}_{2 \mathrm{ML}}+\mathrm{Ad}_{1 \mathrm{ML}}}{2}
\end{aligned}
$$

Equation (4) provides a linear scale, ranging between -1 and 1 and where AS $=0$ indicates perfect gait symmetry (Figure 2c), irrespective of the direction of motion under investigation. Further, while positive symmetry values relate to a gait pattern with a higher regularity of strides compared with steps, negative symmetry values correspond to a gait pattern where the strides are more irregular than the steps. This brings in additional clinical relevance with respect to previous propositions.

In order to investigate the behavior of the three functions when applied to real data, three available datasets were retrospectively analysed (Supplementary Materials). These included data collected from three participant groups within different studies carried out at the Sheffield Teaching Hospitals (which had all received ethical approval from the relevant boards): 24 healthy controls (HC), 20 individuals with Parkinson's Disease (PD), and 20 individuals with multiple sclerosis (MS). These two neurological progressive diseases were chosen for having different aetiologies which reflect in a variety of different clinical symptoms and, from a mobility perspective, in differently impaired gait patterns. The clinical characteristics of the patients within the two cohorts were also quite heterogeneous, as described in Table 1. 
Table 1. Participant Characteristics.

\begin{tabular}{cccc}
\hline Characteristic & 24 HC & 20 PD & 20 MS \\
Sex & 8 males & 14 males & 14 males \\
Age (mean \pm SD) & 16 females & 6 females & 6 females \\
& $50 \pm 8$ years & $59 \pm 7$ years * & $45 \pm 14$ years \\
Disease characteristics & N/A & Average UPDRS-III: $33 \pm 9$ & Average EDSS: $3.7 \pm 1.6$ \\
& & Mild: 9 participants & Mild: 6 participants \\
& & Moderate: 11 participants & Moderate: 3 participants \\
& & Severe: 0 participants & Severe: 11 participants \\
\hline
\end{tabular}

HC: Healthy controls; PD: participants with Parkinson's disease; MS: participants with multiple sclerosis. * Significant difference compared to healthy controls and patients with MS based on a one-way ANOVA. Post-hoc tests were performed with a Bonferroni correction to compare the different groups. Disease characteristics were defined as Mild: UPDRS-III $\leq 32$, Moderate: $33 \leq$ UPDRS-III $\leq 58$, Severe: UPDRS-III $\geq 59$ [6] for PD and Mild: EDSS $\leq 2$, Moderate: $2.5 \leq$ EDSS $\leq 3.5$, Severe: EDSS $\geq 4$ [7].

The data from the MS cohort used in this study is a subset of the data described in Angelini et al. [8]. Twenty participants were selected from that study among those not using walking aids, ensuring that they were sex-matched to the PD cohort. Participants performed a 6-min walk test by walking back and forth on a $10 \mathrm{~m}$ walkway in a hospital corridor at a comfortable speed while wearing triaxial inertial measurement units (IMUs) (OPAL, APDM Inc., Portland, OR, USA, $128 \mathrm{~Hz}$ ). One sensor was placed on the lower back, overlying the fifth lumbar vertebra, with a velcro strap, and two sensors were attached to the left and right shin at the level of the ankle, respectively. The accelerations were reoriented to a horizontal-vertical coordinate system and filtered with a $10 \mathrm{~Hz}$ cut-off, zero phase, low-pass Butterworth filter. Turning phases were identified and isolated using the angular velocity signals [8], so that steady state walking passes could be identified (Figure 1). To be able to compare the same amount of walking passes for each group, the quantity of walking passes completed was defined for each group (HC: 824 passes; MS: 519 passes; PD: 128 passes). The number of passes included was standardized to 128 by taking every 4th pass for MS and every 6th pass for HC. For each of the walking passes $\mathrm{Ad}_{1}$ and $\mathrm{Ad}_{2}$ were calculated as the first two peaks in the autocorrelation function for signal recorded in the $\mathrm{V}, \mathrm{ML}$, and $\mathrm{AP}$ directions and then used to calculate $\mathrm{AS}, \mathrm{S}_{\mathrm{MN}}$, and $\mathrm{AS}_{\mathrm{K}}$.

Normality assumption was checked with the Shapiro-Wilk test and QQ-plots. Since most of the data was not normally distributed, a nonparametric test (Independent-Samples Kruskal-Wallis Test) was used to compare the participant groups. Separate tests were performed for the different metrics $\left(\mathrm{Ad}_{1}, \mathrm{Ad}_{2}, \mathrm{~S}_{\mathrm{MN}}, \mathrm{AS}_{\mathrm{K}}, \mathrm{AS}\right)$ for each axis (AP, ML, V). Pairwise comparisons adjusted by the Bonferroni correction were used as post hoc tests to identify where specific differences occurred between the cohorts. The level of significance was set at 0.05. Calculations were performed using SPSS, version 26 (SPSS; Inc, Chicago, IL, USA). The effect size ( $r$ ) for non-parametric tests was also computed as:

$$
\mathrm{r}=\frac{\mathrm{z}}{\sqrt{\mathrm{N}}}
$$

where $\mathrm{z}$ is the $\mathrm{z}$-score based on the pairwise comparisons, and $\mathrm{N}$ is the number of total observations on which $\mathrm{z}$ is based. Thresholds of $0.1,0.3$, and 0.5 were recommended by Cohen [9] for small, medium, and large effect sizes, respectively. 


\section{Results}

Figure 3 shows the comparison between the three different patient groups for different outcome variables $\left(\mathrm{Ad}_{1}, \mathrm{Ad}_{2}, \mathrm{~S}_{\mathrm{MN}}, \mathrm{AS}_{\mathrm{K}}, \mathrm{AS}\right)$ in the three directions of motion. Table 2 shows the significant differences with accompanying effect sizes for the group comparisons. In the results below, values will be reported as Median [Q1, Q3], where Q1 is the first quartile and Q3 is the third quartile. As immediately visible in the figure, the $\mathrm{AS}_{\mathrm{K}}$ values covered a very broad range of values, especially in the ML direction, where values up to 1000 were observed. This reflected into a substantially different interpretation of the data with respect to both $\mathrm{S}_{\mathrm{MN}}$ and AS when statistically comparing the three different groups.

In the AP direction, lower regularity was observed for both steps and strides in both MS $\left(\mathrm{Ad}_{1}: 0.81[0.65,0.88] ; \mathrm{Ad}_{2}: 0.86\right.$ [0.75, 0.92]) and PD $\left(\mathrm{Ad}_{1}: 0.82\right.$ [0.74, 0.88]; $\left.\mathrm{Ad}_{2}: 0.89[0.83,0.92]\right)$ compared to the $\mathrm{HC}\left(\mathrm{Ad}_{1}: 0.88[0.84,0.91] ; \mathrm{Ad}_{2}: 0.92[0.89,0.94]\right)$ (Figure 3), with no significant differences between the two patient groups. Slightly less asymmetric gait was observed for the HC according to AS (HC: 0.01 [0.00, 0.04], MS: 0.02 [0.01, 0.06], PD: 0.02 [0.00, 0.05]), but these differences were not significant (KruskalWallis $p=0.054$ ). According to $\mathrm{S}_{\mathrm{MN}}$, the MS had lower symmetry than the HC (HC: 0.97 [0.92, 1.00], MS: 0.95 [0.85, 0.98], PD: 0.95 [0.87, 1.00]). The $\mathrm{AS}_{\mathrm{K}}$ values suggested much higher asymmetry for the PD and MS group than for the HC cohort (HC: 0.04 [0.02, 0.09], MS: 0.06 [0.03, 0.17], PD: $0.06[0.03,0.13])$. Again, no significant difference was found between the patient cohorts.

In the ML direction, significant differences were found between the three groups for step and stride regularity, with $\mathrm{HC}$ having the most regular steps $\left(\mathrm{Ad}_{1}:-0.79[-0.85,0.68]\right)$ and strides $\left(\mathrm{Ad}_{2}: 0.86[0.81,0.90]\right)$. Compared to $\mathrm{PD}, \mathrm{MS}$ patients had fewer regular steps $\left(\mathrm{Ad}_{1}, \mathrm{MS}:-0.57[-0.76,-0.54]\right.$ vs PD: $\left.-0.70[-0.80,-0.54]\right)$ and strides $\left(\mathrm{Ad}_{2}, \mathrm{MS}: 0.75\right.$ $[0.58,0.84]$ vs PD: 0.82 [0.71, 0.87]). For gait symmetry, $\mathrm{S}_{\mathrm{MN}}$ values suggested higher symmetry for the $\mathrm{HC}$, but no significant differences were found between the three groups (HC: -0.93 [-0.98, -0.76]; MS: -0.90 [-1.00, -0.70]; PD: -0.89 [-0.97, -0.76], KruskalWallis: $p=0.119$ ). $\mathrm{AS}_{\mathrm{K}}$ values had a much larger range in the ML direction than in AP or $\mathrm{V}$ and as a result indicated that the $\mathrm{HC}$ had a much higher level of asymmetry (34.10 $[13.55,100.84])$ than both PD $(17.24[5.59,44.35])$ and MS patients $(11.20[3.96,38.94])$, but the differences between the latter two groups remained non-significant due to the excessively large variability within the groups. The AS value indicated no significant differences between the three cohorts (HC: 0.05 [0.01, 0.10]; MS: 0.04 [0.00, 0.10]; PD: 0.05 [0.01, 0.10], Kruskal-Wallis: $p=0.152)$.

In the $\mathrm{V}$ direction, both MS $\left(\mathrm{Ad}_{1}: 0.85[0.73,0.93]\right)$ and $\mathrm{PD}\left(\mathrm{Ad}_{1}: 0.89[0.82,0.92]\right)$ had significantly lower step regularity compared to $\mathrm{HC}\left(\mathrm{Ad}_{1}: 0.94[0.90,0.96]\right)$, but no significant differences were observed between the two patient groups. Significant differences were found between the three groups for stride regularity, with $\mathrm{HC}\left(\mathrm{Ad}_{2}: 0.96[0.93,0.97]\right)$ having the most regular strides, followed by PD $\left(\mathrm{Ad}_{2}: 0.93[0.90,0.96]\right)$ and $\mathrm{MS}\left(\mathrm{Ad}_{2}: 0.90\right.$ $[0.77,0.95])$. When symmetry was calculated by $\mathrm{S}_{\mathrm{MN}}$, the PD cohort ( $\left.\mathrm{S}_{\mathrm{MN}}: 0.96[0.88,1.00]\right)$ appeared to be less symmetrical than the $\mathrm{HC}\left(\mathrm{S}_{\mathrm{MN}}: 0.99[0.96,1.00]\right)$ and the MS cohorts ( $\left.\mathrm{S}_{\mathrm{MN}}: 0.98[0.93,1.01]\right)$. Meanwhile, $\mathrm{AS}_{\mathrm{K}}$ values indicated that there were differences between HC $(0.02[0.01,0.04])$ and both MS $(0.04[0.02,0.10])$ and PD $(0.05[0.02,0.13])$, but not between the two patient groups. AS values were closer to the $\mathrm{S}_{\mathrm{MN}}$ findings and significant differences between cohorts were also observed (Kruskall Wallis $p<0.001$ ) with HC (AS: 0.01 [0.00, 0.02]) having lower values than MS (AS: 0.01 [0.00, 0.02]) and PD (AS: $0.02[0.00,0.05])$. 


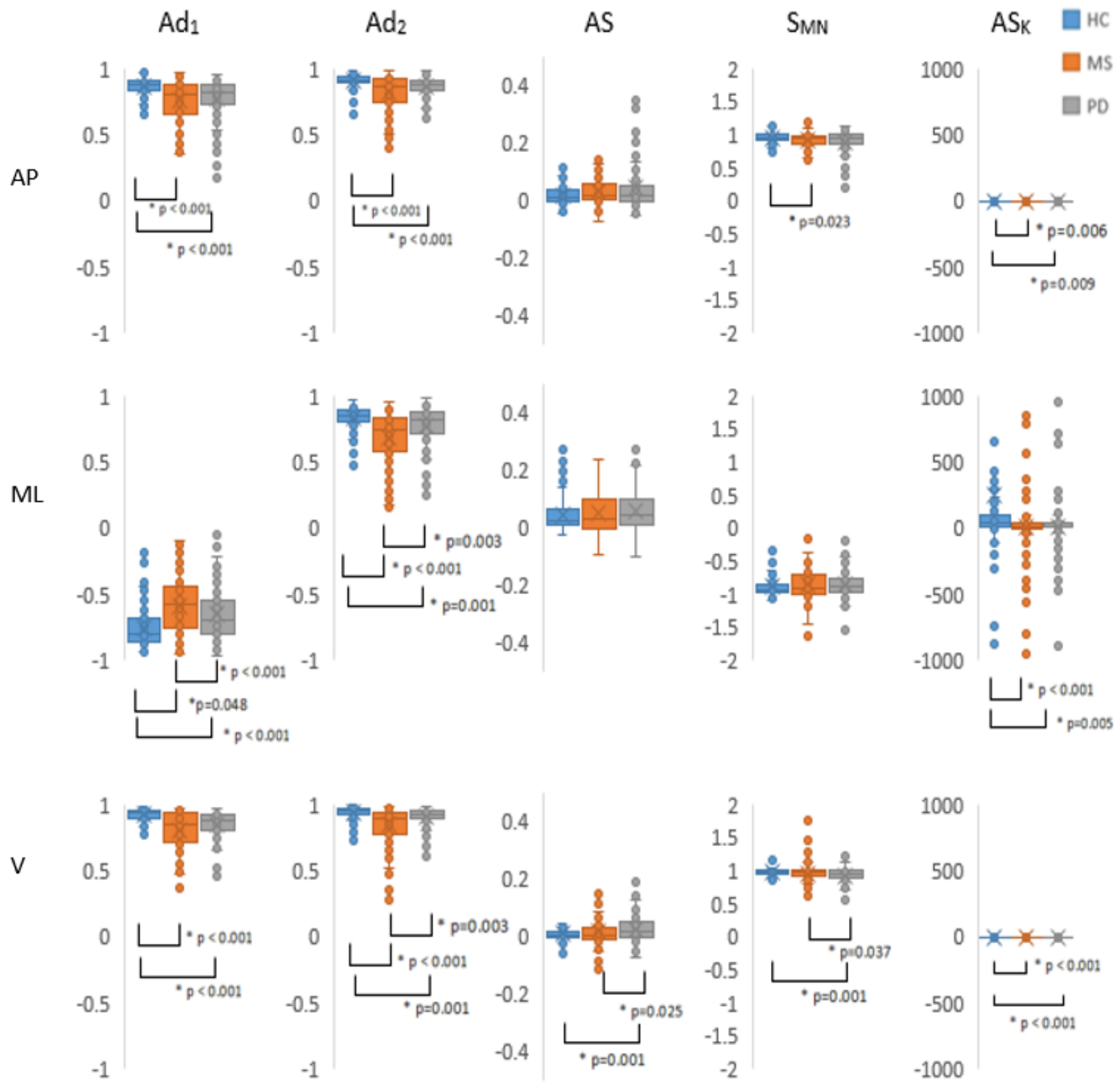

Figure 3. Comparison between the three different patient groups for different outcome variables $\left(\operatorname{Ad}_{1}, \mathrm{Ad}_{2}, \mathrm{AS}, \mathrm{S}_{\mathrm{MN}}, \mathrm{AS}_{\mathrm{K}}\right)$. *: significant difference between groups. $\mathrm{Ad}_{1}$ : step regularity; $\mathrm{Ad}_{2}$ : stride regularity; $\mathrm{AS}$ : Asymmetry as proposed in this article; $\mathrm{S}_{\mathrm{MN}}$ : symmetry as proposed by Moe-Nilssen and Helbostad [3]; $\mathrm{AS}_{\mathrm{K}}$ : Asymmetry as proposed by Kobsar et al. [2]; AP: anterior-posterior direction; ML: medial-lateral direction; V: vertical direction. 
Table 2. $p$-values and effect sizes for comparisons between groups.

\begin{tabular}{|c|c|c|c|c|c|c|}
\hline Direction & Comparison & $\mathbf{A d}_{1}$ & $\mathrm{Ad}_{2}$ & AS & $\mathrm{S}_{\mathrm{MN}}$ & $\mathbf{A S}_{\mathbf{K}}$ \\
\hline \multirow[t]{7}{*}{$\mathrm{AP}$} & Group & $p<0.001 *$ & $p<0.001 *$ & $p=0.054$ & $p=0.022 *$ & $p=0.002 *$ \\
\hline & HC-MS & $p<0.001^{*}$ & $p<0.001$ & na & $p=0.023$ & $p=0.006$ \\
\hline & & $\mathrm{r}=-0.30(\mathrm{~m})$ & $\mathrm{r}=-0.31(\mathrm{~m})$ & & $\mathrm{r}=-0.14(\mathrm{~s})$ & $\mathrm{r}=-0.16(\mathrm{~s})$ \\
\hline & HC-PD & $p<0.001 *$ & $p<0.001$ & na & $p=0.138$ & $p=0.009$ \\
\hline & & $\mathrm{r}=-0.27(\mathrm{~s})$ & $\mathrm{r}=0.23(\mathrm{~s})$ & & $\mathrm{r}=-0.10(\mathrm{~s})$ & $\mathrm{r}=-0.15(\mathrm{~s})$ \\
\hline & MS-PD & $p=1.000$ & $p=0.371$ & na & $p=1.000$ & $p=1.000$ \\
\hline & & $r=-0.02$ & $r=-0.08$ & & $r=-0.03$ & $r=-0.01$ \\
\hline \multirow[t]{7}{*}{ ML } & Group & $p<0.001 *$ & $p<0.001 *$ & $p=0.152$ & $p=0.119$ & $p<0.001 *$ \\
\hline & HC-MS & $p<0.001^{*}$ & $p<0.001$ & & & $p<0.001$ \\
\hline & & $\mathrm{r}=-0.33(\mathrm{~m})$ & $\mathrm{r}=-0.35(\mathrm{~m})$ & na & na & $r=-0.23(\mathrm{~s})$ \\
\hline & HC-PD & $p<0.001 *$ & $p=0.001$ & & & $p=0.005$ \\
\hline & & $\mathrm{r}=-0.20(\mathrm{~s})$ & $\mathrm{r}=-0.19(\mathrm{~s})$ & na & na & $\mathrm{r}=-0.16(\mathrm{~s})$ \\
\hline & MS-PD & $p=0.048^{*}$ & $p=0.003$ & & & $p=0.588$ \\
\hline & & $\mathrm{r}=-0.12(\mathrm{~s})$ & $r=-0.17$ & na & na & $r=-0.07$ \\
\hline \multirow[t]{7}{*}{$\mathrm{V}$} & Group & $p<0.001^{*}$ & $p<0.001^{*}$ & $p=0.001 *$ & $p=0.001 *$ & $p<0.001 *$ \\
\hline & HC-MS & $p<0.001 *$ & $p<0.001$ & $p=0.849$ & $p=0.675$ & $p<0.001$ \\
\hline & & $\mathrm{r}=-0.40(\mathrm{~m})$ & $\mathrm{r}=-0.40(\mathrm{~m})$ & $\mathrm{r}=-0.05$ & $r=-0.06$ & $\mathrm{r}=-0.23(\mathrm{~s})$ \\
\hline & HC-PD & $p<0.001 *$ & $p<0.001$ & $p=0.001$ & $p=0.001$ & $p<0.001$ \\
\hline & & $\mathrm{r}=-0.36(\mathrm{~m})$ & $\mathrm{r}=-0.23(\mathrm{~s})$ & $\mathrm{r}=-0.19(\mathrm{~s})$ & $\mathrm{r}=-0.19(\mathrm{~s})$ & $\mathrm{r}=-0.26(\mathrm{~s})$ \\
\hline & MS-PD & $p=1.000$ & $p=0.003$ & $p=0.025$ & $p=0.037$ & $p=1.000$ \\
\hline & & $r=-0.04$ & $\mathrm{r}=-0.17(\mathrm{~s})$ & $\mathrm{r}=-0.13(\mathrm{~s})$ & $\mathrm{r}=-0.13(\mathrm{~s})$ & $\mathrm{r}=-0.04$ \\
\hline
\end{tabular}

$\mathrm{Ad}_{1}$ : step regularity; $\mathrm{Ad}_{2}$ : stride regularity; AS: Asymmetry as proposed in this article; $\mathrm{S}_{\mathrm{MN}}$ : symmetry as proposed by Moe-Nilssen and Helbostad [3]; $\mathrm{AS}_{\mathrm{K}}$ : Asymmetry as proposed by Kobsar et al. [2]; AP: anterior-posterior direction; ML: medial-lateral direction; V: vertical direction; $p=p$-value, ${ }^{*}$ significant difference $(p<0.05)$; r: effect size; (s): small effect size, (m): moderate effect size; (l): large effect size; na= not applicable, due to no significant effect for the Independent-Samples Kruskal-Wallis Test.

\section{Discussion}

The aim of this paper was to propose a linear and easy to interpret quantification of gait asymmetry and illustrate how this new calculation could facilitate the comparison between observations related to the different directions of motion and the clinical interpretation of the data. Previous quantifications of gait (a)symmetry, $S_{M N}$ and $\mathrm{AS}_{\mathrm{K}}$, implement discontinuous scales potentially ranging to infinity which hinders interpretation of results. The proposed asymmetry metric, conversely, is a continuous and linear scale varying between -1 and 1 , with only one value $(A S=0)$ indicating perfect symmetry. In this way it is possible to obtain measures of gait asymmetry that are immediately comparable. In addition, the proposed scale allows the immediate identification of whether asymmetries originate from differences in step or stride regularity. All these aspects make highlighting and interpreting differences between the gait patterns of different participants more straightforward.

One of the main complexities of previous definitions was associated with their application to the analysis of the data measured in the ML direction. In this direction, negative values of $\mathrm{Ad}_{1}$, would contribute to negative values of both $\mathrm{S}_{\mathrm{MN}}$ and $\mathrm{AS}_{\mathrm{K}}$, which would eventually cause discontinuity in their values. To compensate for this issue, even if not explicitly stated in their paper, it might be assumed from the reported results that in Kobsar et al. [2], $\mathrm{Ad}_{1}$ was included as an absolute value in the calculation of $\mathrm{AS}_{\mathrm{K}}$ in the ML direction. This would explain the large differences between the values reported here and those observed for their young $(15.34 \pm 18.52)$ and older $(15.42 \pm 19.61)$ participants. The same would apply to the results of Barden et al. [10], who reported positive values for $\mathrm{Ad}_{1}$, both for healthy controls and patients with knee osteoarthritis. As confirmed by the author, Angelini et al. [8], took the absolute value of $\mathrm{Ad}_{1}$ in the ML direction when calculating $\mathrm{AS}_{\mathrm{K}}$ and then plotted the inverse values to facilitate comparison with the other directions. Our proposed calculation contains an explicit reference to the different calculation for the different directions and will hence allow for more straightforward comparisons between results in future studies. 
To investigate the effects that different calculations would have on the clinical interpretation of the data, we tested the behavior of the various methods in discriminating between three patient groups. While consistent results were observed in all directions when calculating symmetry using $\mathrm{S}_{\mathrm{MN}}$ and $\mathrm{AS}, \mathrm{AS}_{\mathrm{K}}$ indicated a different interpretation. In particular, $\mathrm{AS}_{\mathrm{K}}$ provided a much larger range of values calculated for AS. There were also large disparities between data from different signal axes, with ranges of values in the ML direction being up to 1000 times bigger than those in the AP and V directions. This would make it virtually impossible to compare results from individual patients. Despite its consistency with AS in terms of between groups comparison, the complexity of interpretation of non-linear data still needs to be considered for $\mathrm{S}_{\mathrm{MN}}$.

In the present study, we were able to show the analytical flows of current methods and highlight the risks of data misinterpretation that could stem from a lack of consistency of the results obtained from the various calculations. In line with previous literature (Angelini et al. [5]; Buckley et al. [4]), our results indicate that while step and stride regularity have a clear potential as biomarkers of disease progression in MS and PD, step asymmetry seems to be less relevant for these patients. While it might be hypothesized that the proposed index could be sensitive to gait impairments when investigating cohorts with highly asymmetric walking, such as for example hemiplegic patients or those who had a hip replacement, additional investigations would be needed to prove this hypothesis.

This study has its clear limitations. First of all, as the size of the cohorts were limited and the two groups were highly heterogeneous, generalisability and clinical interpretability of the findings are also limited. Also, this hinders the possibility of establishing a clear cause-effect relationship with respect to disease progression. More importantly, a full validation of the clinical meaning of the index would require data to be simultaneously collected with a gold standard such as an optoelectronic system. This would allow further investigatation of the link between specific impairments and motion, and evaluation of its sensitivity to change.

\section{Conclusions}

This study proposed a linear index for the quantification of gait symmetry and illustrated how this overcomes the limitation of previous definitions. The new index provides comparable and interpretable data in all directions of motion.

Supplementary Materials: The following are available online at https://www.mdpi.com/article/10 .3390/sym13091560/s1, Excel file: data.

Author Contributions: Conceptualization, L.M.A.v.G. and C.M.; Data curation, L.M.A.v.G., L.A. and E.E.B.; Formal analysis, L.M.A.v.G., L.A. and E.E.B.; Funding acquisition, C.M.; Investigation, L.A. and E.E.B.; Methodology, L.M.A.v.G., L.A., E.E.B. and C.M.; Project administration, L.A., E.E.B. and C.M.; Resources, C.M.; Software, L.M.A.v.G. and E.E.B.; Supervision, C.M.; Validation, L.A. and E.E.B.; Visualization, L.M.A.v.G.; Writing-original draft, L.M.A.v.G.; Writing-review \& editing, L.A., E.E.B. and C.M. All authors have read and agreed to the published version of the manuscript.

Funding: This study was co-funded by the NIHR through the Sheffield Biomedical Research Centre (BRC, grant number IS-BRC-1215-20017), by the European Union's Horizon 2020 research and innovation programme and EFPIA via the Innovative Medicine Initiative 2 (Mobilise-D project, grant number IMI22017-13-7-820820), and the UK Engineering and Physical Sciences Research Council (Multisim and MultiSim2 projects, grant numbers EP/K03877X/1 and EP/S032940/1, respectively). The views expressed are those of the author(s) and not necessarily those of the NHS, the NIHR, the Department of Health and Social Care, the IMI, the European Union, the EFPIA, or any Associated Partners. This study was carried out at the NIHR Sheffield Clinical Research Facility. We would like to thank all participants for giving their time to support this research.

Institutional Review Board Statement: These studies were conducted according to the guidelines of the Declaration of Helsinki, and approved by the relevant Ethics Committees. Ursodeoxycholic acid as a novel disease-modifying treatment for Parkinson's disease, The 'UP' study (East of EnglandCambridgeshire and Hertfordshire; 18/EE/0280; Approval Date: 18/11/2018). Multiple Sclerosis- 
Secondary Progressive Multi-Arm Randomisation Trial, MS-SMART (Scotland A; 13/SS/0007; Approval Date: $11 / 01 / 2013)$. Advanced inertial sensors to quantify disability progression in patients with secondary progressive Multiple Sclerosis taking part in the MS SMART clinical trial, AIMS (Bradford Leeds; 15/YH/0300; Approval Date: 03/09/2015).

Informed Consent Statement: Written informed consent was obtained from all subjects involved in the study.

Data Availability Statement: The data presented in this study are available in the supplementary material.

Conflicts of Interest: The funders had no role in the design of the study; in the collection, analyses, or interpretation of data; in the writing of the manuscript, or in the decision to publish the results.

\section{References}

1. Viteckova, S.; Kutilek, P.; Svoboda, Z.; Krupicka, R.; Kauler, J.; Szabo, Z. Gait symmetry measures: A review of current and prospective methods. Biomed. Signal Process. Control 2018, 42, 89-100. [CrossRef]

2. Kobsar, D.; Olson, C.; Paranjape, R.; Hadjistavropoulos, T.; Barden, J.M. Evaluation of age-related differences in the stride-to-stride fluctuations, regularity and symmetry of gait using a waist-mounted tri-axial accelerometer. Gait Posture 2014, 39, 553-557. [CrossRef] [PubMed]

3. Moe-Nilssen, R.; Helbostad, J.L. Estimation of gait cycle characteristics by trunk accelerometry. J. Biomech. 2004, 37, 121-126. [CrossRef]

4. Buckley, C.; Galna, B.; Rochester, L.; Mazzà, C. Upper body accelerations as a biomarker of gait impairment in the early stages of Parkinson's disease. Gait Posture 2019, 71, 289-295. [CrossRef] [PubMed]

5. Angelini, L.; Buckley, E.; Bonci, T.; Radford, A.; Sharrack, B.; Paling, D.; Nair, K.P.S.; Mazza, C. A Multifactorial Model of Multiple Sclerosis Gait and its Changes Across Different Disability Levels. IEEE Trans. Biomed. Eng. 2021. [CrossRef] [PubMed]

6. Martínez-Martín, P.; Rodríguez-Blázquez, C.; Alvarez, M.; Arakaki, T.; Arillo, V.C.; Chaná, P.; Fernández, W.; Garretto, N.; Martínez-Castrillo, J.C.; Rodríguez-Violante, M.; et al. Parkinson's disease severity levels and MDS-Unified Parkinson's Disease Rating Scale. Park. Relat. Disord. 2015, 21, 50-54. [CrossRef] [PubMed]

7. Kurtzke, J.F. Rating neurologic impairment in multiple sclerosis: An expanded disability status scale (EDSS). Neurology 1983, 33, 1444-1452. [CrossRef] [PubMed]

8. Angelini, L.; Hodgkinson, W.; Smith, C.; Dodd, J.M.; Sharrack, B.; Mazzà, C.; Paling, D. Wearable sensors can reliably quantify gait alterations associated with disability in people with progressive multiple sclerosis in a clinical setting. J. Neurol. 2020, 267, 2897-2909. [CrossRef]

9. Cohen, J. CHAPTER 3-The Significance of a Product Moment rs. In Statistical Power Analysis for the Behavioral Sciences; Cohen, J., Ed.; Academic Press: Cambridge, MA, USA, 1977; pp. 75-107. [CrossRef]

10. Barden, J.M.; Clermont, C.A.; Kobsar, D.; Beauchet, O. Accelerometer-based step regularity is lower in older adults with bilateral knee osteoarthritis. Front. Hum. Neurosci. 2016, 10, 625. [CrossRef] 